
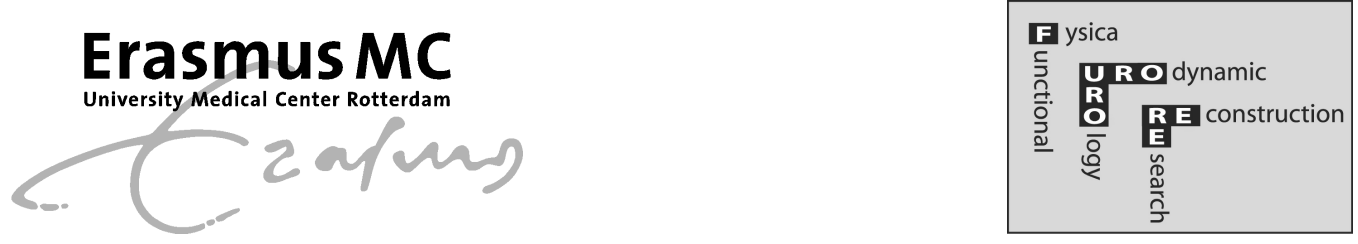

\title{
Is the impaired flow after hypospadias correction due to increased urethral stiffness?
}

\section{Authors:}

Tim Idzenga ${ }^{1}$, Dik J. Kok ${ }^{2}$, Johan J.M. Pel ${ }^{1}$, Ron van Mastrigt ${ }^{1}$ and Katja P. Wolffenbuttel ${ }^{2}$

\section{Institute:}

${ }^{1}$ Dept. of Urology, sector Furore, Erasmus MC Rotterdam, The Netherlands

2 Dept. of Paediatric Urology, Erasmus MC Rotterdam, The Netherlands

\section{Correspondence to:}

Tim Idzenga

Erasmus MC, dept. of Urology

Sector Furore, room EE1630

PO Box 1738

3000 DR, Rotterdam, The Netherlands

Tel: **-31-10-4087379

Fax: **-31-10-4089451

t.idzenga@erasmusmc.nl 


\begin{abstract}
Purpose: A low flow rate without clinical symptoms is commonly found in boys after hypospadias correction. Urethral calibration usually shows no abnormalities. We investigated whether this flow rate impairment might be caused by increased neo-urethral wall-stiffness. Methods: From Polyvinyl Alcohol cryogel two models of the urethra were made, a hypospadias model and a control model. Both models had a constant and equal inner diameter and equal compliance. The hypospadias model had a less compliant distal segment mimicking the distal neo-urethra after hypospadias correction. In both models flow rate was recorded as a function of bladder pressure. To test whether the length of the less compliant segment had an effect on the flow rate, both models were shortened by cutting off $1 \mathrm{~cm}$ segments. Results: In a physiological range of bladder pressures $\left(10-130 \mathrm{~cm} \mathrm{H}_{2} \mathrm{O}\right)$ the mean flow rate $( \pm 1 \mathrm{sem})$ in the hypospadias model was $2.8 \pm 0.3 \mathrm{ml} / \mathrm{s}$, significantly lower $(\mathrm{p}<.05)$ than in the control model $(5.4 \pm 0.6 \mathrm{ml} / \mathrm{s})$. Shortening of the hypospadias model showed some increase in flow rate, however not statistically significant. In the control model there was also no significant variation in flow rate. Conclusion: We showed that a low compliant segment of a urethral model reduces the flow rate. Extrapolating these results to asymptomatic boys with a low urinary flow rate after hypospadias repair might justify a watchful waiting policy.
\end{abstract}




\section{Introduction}

Hypospadias is a congenital malformation with an incidence of up to $0.73 \%$ in the general population (Pierik et al. 2002). Treatment and follow up of hypospadias patients forms a large part of the work of pediatric urologists. Surgical repair of hypospadias comprises lengthening of the urethra to obtain a normally placed meatus, chordee correction and remodelling of the abnormal penile skin. Probably the most frequently used technique for the correction of hypospadias is the tubularized incised plate correction (TIP or Snodgrass procedure (Snodgrass 1994)). In the short term complications like fistula and urethral stricture formation occur in 5\% (primary cases (Borer et al. 2001)) to 17.5\% (proximal hypospadias (Chen et al. 2000)) of the cases. An impaired flow rate is often observed in asymptomatic boys after hypospadias correction. A urethral stricture is, however, rarely found. An open question is whether the neo-urethra will function adequately throughout adult life.

Recently we reported on the urine flow characteristics of boys with hypospadias before and after correction of hypospadias (Wolffenbuttel et al. 2005). The cause of the impaired flow rate in hypospadias patients is unknown. Two factors might play a role in this impaired flow rate: narrowing of the urethral lumen or stricture (intraluminal factor) and diminished compliance (i.e. increased stiffness) of the urethral wall or the surrounding tissue surrounding (extraluminal factor). In this study we tested the hypothesis that an impaired urinary flow rate may be caused by a low compliance of the neo-urethral wall. We therefore constructed two models of the urethra from Polyvinyl Alcohol: one model in which the distal segment had low compliance, mimicking a urethra after hypospadias correction, and one control model (see Figure 1). We measured the flow rate in the hypospadias model and compared this to the flow rate in the control model. We also tested the influence of the length of the low compliant segment on the flow rate. 


\section{Materials \& Methods}

We constructed 2 model urethras from Polyvinyl Alcohol (PVA) cryogel. Repeated freezing and thawing of the PVA controls its elastic properties. With increasing number of freeze/thaw cycles the stiffness of the PVA increases. We made the models by pouring a $10 \%$ aqueous solution of PVA into a cylindrical mould ( $400 \mathrm{~mm}$ in length, $16 \mathrm{~mm}$ in diameter (Idzenga, Pel, Baldewsing \& Mastrigt 2005)). To create a part with low compliance we placed a circular rod along the central axis with a diameter of $4 \mathrm{~mm}$ and poured PVA (mixed with Carborundum particles of 3-10 $\mu \mathrm{m}$ for contrast between stiff and normal tissue) into the mould until approximately $3 \mathrm{~cm}$ of the mould was filled. After one freeze-thaw cycle we added a segment of approximately

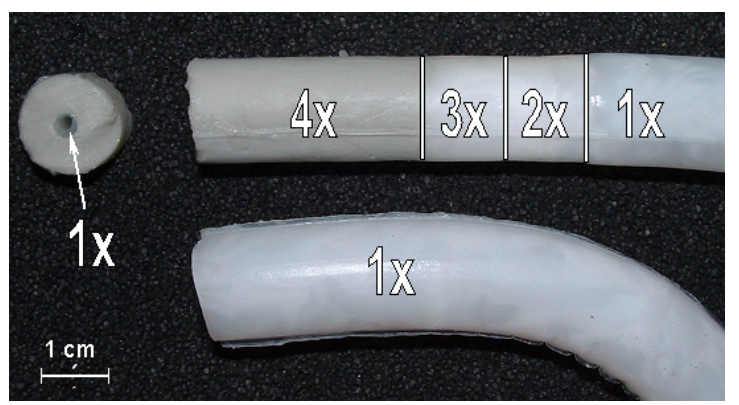

Figure 1: Model of the human male urethra after hypospadias correction (upper model) and control model (bottom model), both made from Polyvinyl Alcohol. On the left a cross section is shown of the hypospadias model. This model had a segment (grey) with diminished compliance (freeze-thawed four times) and two segments that were freeze-thawed three or two times mimicking the neo-urethra. The control model had uniform compliance (freeze-thawed once).

$1 \mathrm{~cm}$ length and once again freeze-thawed the mould. We added another $1 \mathrm{~cm}$ segment of PVA and again repeated the freeze-thaw cycle. After these three freezethaw cycles we removed the $4 \mathrm{~mm}$ diameter rod and replaced it with a rod with a diameter of $2 \mathrm{~mm}$. We completely filled up the mould with PVA and repeated the freeze-thaw cycle once more. We removed the model from the mould and cut off excess PVA resulting in a model urethra freeze-thawed once (Elastic modulus $\sim 2$ $\mathrm{x} 10^{4} \mathrm{~N} / \mathrm{m}^{2}$ ) with a total length of $30 \mathrm{~cm}$ including a $3 \mathrm{~cm}$ long distal segment that was freeze-thawed four times (Elastic modulus $\sim 5.9 \times 10^{4} \mathrm{~N} / \mathrm{m}^{2}$ ) and two $1 \mathrm{~cm}$ long parts that were freeze-thawed respectively three and two times. To obtain a control model we cut off $15 \mathrm{~cm}$ of the once freeze-thawed segment. This resulted in two models of $15 \mathrm{~cm}$ length each with equal stiffness except for the distal segment of the hypospadias model (see Figure 1). Both models had an inner diameter of 2 $\mathrm{mm}$. 


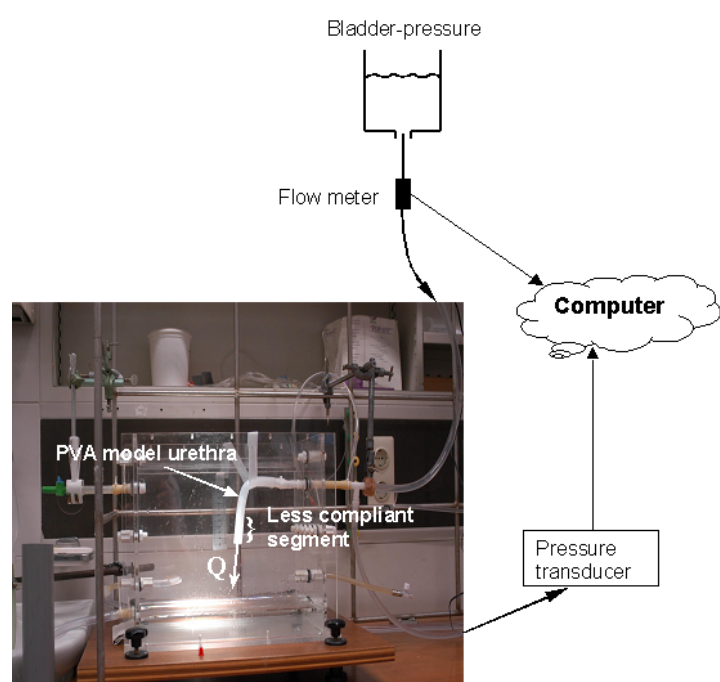

Figure 2: Measurement setup for recording of flow through the hypospadias model and the control model. Both were placed in a custom made container and connected via a turbine flow meter to a water column. The bladder pressure was recorded at the entrance of the container.
Each model was placed in a custommade container (Idzenga et al. 2005). Polyurethane tubing was used to connect the models via a turbine flow meter (Flosensor 101, McMillan Company, USA) to a water column representing the bladder. The water level in this column was kept constant using a floating device to maintain a constant pressure. To exclude errors due to the pressure loss caused by connective tubing we measured the resulting bladder pressure with a pressure transducer at the entrance of the container (see Figure 2). The measured bladder pressure and the resulting flow rate were recorded on a personal computer using an A/Dconverter (PCI-6221, National Instruments, Woerden, The Netherlands) in combination with a custom-written Labview-program (National Instruments, Woerden, The Netherlands).

We applied increasing levels of bladder pressure to both models and measured the resulting flow rate. We increased the level of the water column from 10 to 170 $\mathrm{cm} \mathrm{H}_{2} \mathrm{O}$ in steps of $10 \mathrm{~cm} \mathrm{H}_{2} \mathrm{O}$. Due to pressure loss in the turbine flow meter and the connective tubing this resulted in a bladder pressure range from 10 to 130 $\mathrm{cm} \mathrm{H}_{2} \mathrm{O}$. To test the influence of the length of the least compliant segment on the flow rate we decreased the length of both models from 15 to $14,13,12.5$ and 12 $\mathrm{cm}$ by cutting off distal sections. At each length we repeated the pressure flow measurements.

In each set of pressure flow measurements we calculated the mean ( $\pm 1 \mathrm{sem})$ flow rate at the bladder pressure values between 10 and $130 \mathrm{~cm} \mathrm{H}_{2} \mathrm{O}$. At each length of the models we compared the mean flow rates between the two models using an independent samples t-test. We tested whether the length of the least compliant segment affected the mean flow rate using Analysis of Variance. 


\section{Results}

Figure 3 shows the urine flow rate in the two models as a function of the applied bladder pressure at maximum length of the models. In the hypospadias model the mean $( \pm$ 1 sem) flow rate $(2.8 \pm 0.3 \mathrm{ml} / \mathrm{s})$ was significantly lower $(\mathrm{p} ;$.05) than the mean flow rate in the control model $(5.4 \pm 0.6 \mathrm{ml} / \mathrm{s})$. The measured bladder pressures in the control model are shifted to the left relatively to the hypospadias model. This shift is due to the increased pressure loss in turbine flow meter and connective tubing caused by the higher flow rate in the control model.

The mean $( \pm 1$ sem) flow rates through both models are plotted in Figure 4 as a function of the length of the models. At 15, 14 and $13 \mathrm{~cm}$ there was a significant difference in flow rate between the hypospadias and the control model. Shortening of both models did

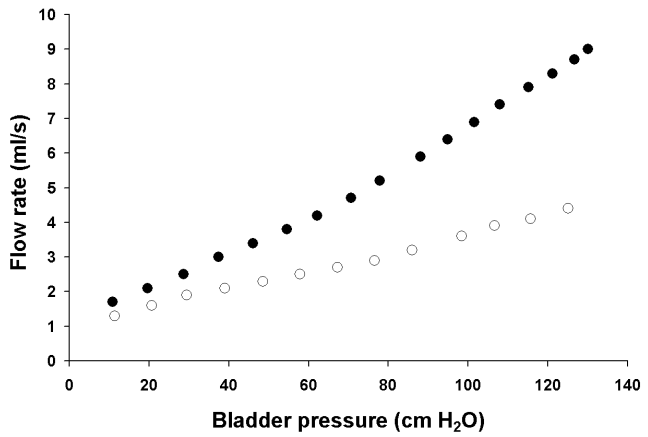

Figure 3: Flow rate through the two model urethras $(\mathrm{O}=$ hypospadias model, $\bullet$ $=$ control model) as a function of the applied bladder pressure. Due to the higher flow rate in the control model the bladder pressure values in the control model are shifted to the left with respect to the bladder pressure values in the hypospadias model. This is caused by increased pressure loss over the connective tubing and the turbine flow meter at the higher flow rate. not affect the flow rate significantly. In the hypospadias model the flow rate seemed to increase a little when the least compliant segment (freeze-thawed four times) was shortened to approximately $0.5 \mathrm{~cm}$, but this increase was not significantly different $(p=.973)$. In the control model the flow rate did not increase upon shortening of the model $(\mathrm{p}=.998)$.

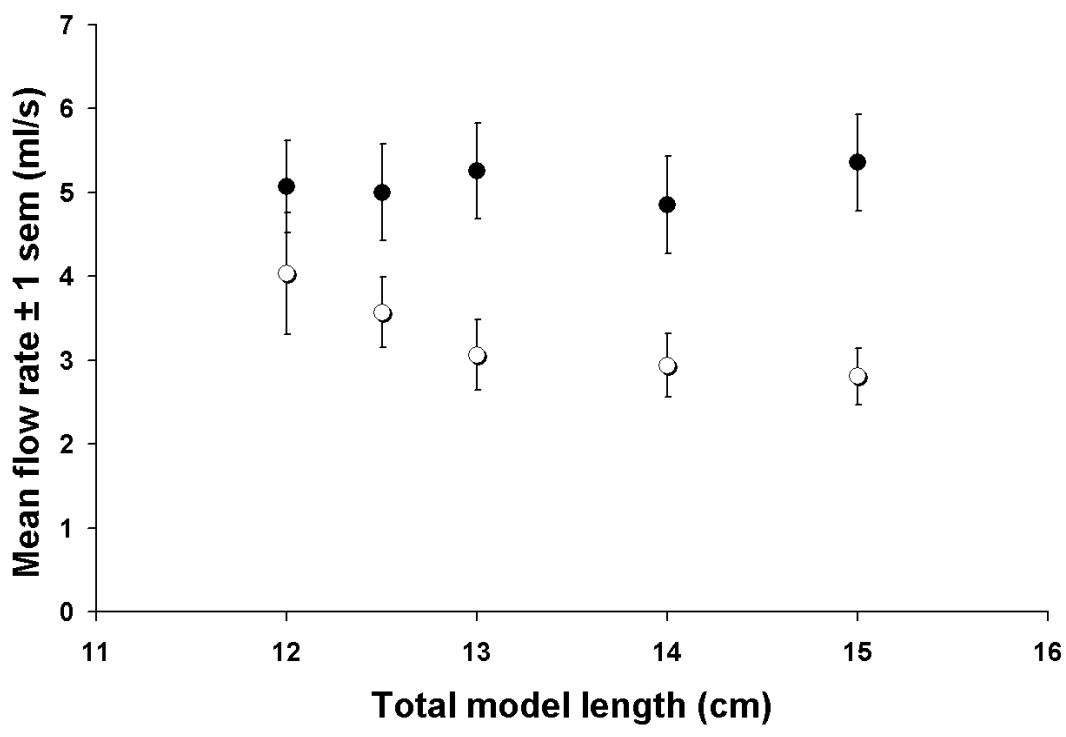

Figure 4: Mean ( $\pm 1 \mathrm{sem}$ ) flow rate between 10 and $130 \mathrm{~cm} \mathrm{H}_{2} \mathrm{O}$ bladder pressure as a function of the length of the model $(0=$ hypospadias model, $\bullet=$ control model). At the lengths marked $*$ the difference is significant at the .05 level. 


\section{Discussion}

We constructed a model urethra with a low compliant distal segment, mimicking a male urethra with low compliance of the urethral wall after hypospadias correction and a control model mimicking a normally developed male urethra. The compliance of the used PVA decreased with increasing number of freeze-thaw cycles. The control model was freeze-thawed once, whereas the hypospadias model was freeze-thawed once with a distal segment that was freeze-thawed four times. The added segments of $1 \mathrm{~cm}$ length in the hypospadias model were necessary to firmly connect the once freeze-thawed segment to the four times freeze-thawed segment. Both models had equal inner diameter. The pressure - flow measurements in the two models showed that the mean flow rate in the hypospadias model with the low compliant distal segment was significantly lower than the flow rate in the control model (see Figure 4). They also showed that a decrease in length of the low compliant segment did not have a significant effect on the flow rate. In general, these results show that low compliance of a segment of the urethral wall is able to diminish the flow rate.

The lower flow rate in a urethra with a segment of low compliance can be explained as follows. The pressure generated in the bladder by contraction of the detrusor muscle drives the urine through the urethra. To this end the urine opens the urethra. When the urethral wall is more compliant it can be opened wider, i.e. to a larger cross sectional area. Consequently at a given bladder pressure the urethra can accommodate a larger flow rate. When a segment of the urethra has lower compliance it is opened less wide, i.e. to a smaller cross sectional area. Therefore a urethra with a less compliant segment can accommodate a lower flow rate at the same given bladder pressure. Shortening the least compliant segment of the hypospadias model had no significant effect on the flow rate. When the length of the distal segment approached zero a small increase in flow rate was observed. This small increase in flow rate is due to the increase in compliance. With the increase in compliance the hypospadias model can accommodate a larger flow rate.

Whether the compliance of the newly constructed urethra after hypospadias correction is actually lower than normal remains to be established. Two possible mechanisms could diminish the compliance. The first is fibrosis around the suture line of the new urethra. The excess collagen or the scar tissue increases the stiffness of the new urethral wall resulting in diminished compliance in comparison to a normally developed urethra. Careful histological analysis of the urethral wall after a TIP procedure in an animal model, however, did not show excess collagen deposition or scarring (Lopes et al. 2001). A second possible mechanism is lack of tissue development around the new urethra resulting in lower compliance of the surrounding tissue than normal. Elastic fibers are a major component of the extracellular matrix of this surrounding tissue (Bastos et al. 2004). In this recent study the authors hypothesised that in utero biomechanical processes such as voiding, erection and amniotic pressure are closely related to development of the foetal urethral extracellular matrix. As the new urethra after hypospadias correction was not exposed before to these biomechanical processes this may explain the lack of compliance.

In conclusion we have shown in our hypospadias model that lack of compliance 
of a segment of the urethra results in a low flow rate. The length of the low compliant segment had little to no effect on the flow rate. Extrapolated to patients after hypospadias correction, an impaired flow rate could be caused by low compliance of the newly constructed urethra due to a local aberration of the surrounding tissue. The length of this low compliant newly constructed urethra does not affect the flow rate significantly. Thus, when an impaired urinary flow rate is observed in asymptomatic boys after hypospadias correction low compliance of the newly constructed urethra has to be considered, warranting a watchful waiting policy.

\section{Acknowledgement}

This research was supported by the Technology Foundation STW, applied division of the Netherlands Organization for Scientific Research (NWO) and the technology programme of the Ministry of Economic Affairs.

\section{References}

Bastos, A. L., Silva, E. A., Silva Costa, W. \& Sampaio, F. J. (2004), 'The concentration of elastic fibres in the male urethra during human fetal development', BJU Int 94(4), 620-3.

Borer, J. G., Bauer, S. B., Peters, C. A., Diamond, D. A., Atala, A., Cilento, B. G., J. \& Retik, A. B. (2001), 'Tubularized incised plate urethroplasty: expanded use in primary and repeat surgery for hypospadias', J Urol 165(2), 581-5.

Chen, S. C., Yang, S. S., Hsieh, C. H. \& Chen, Y. T. (2000), 'Tubularized incised plate urethroplasty for proximal hypospadias', BJU Int 86(9), 1050-3.

Idzenga, T., Pel, J., Baldewsing, R. A. \& Mastrigt, R. (2005), 'Perineal noise recording as a non-invasive diagnostic method of urinary bladder outlet obstruction: a study in polyvinyl alcohol and silicone model urethras', Neurourol Urodyn 24(4), 381-8.

Lopes, J. F., Schned, A., Ellsworth, P. I. \& Cendron, M. (2001), 'Histological analysis of urethral healing after tubularized incised plate urethroplasty', $J$ Urol 166(3), 1014-7.

Pierik, F. H., Burdorf, A., Nijman, J. M., de Muinck Keizer-Schrama, S. M., Juttmann, R. E. \& Weber, R. F. (2002), 'A high hypospadias rate in the netherlands', Hum Reprod 17(4), 1112-5.

Snodgrass, W. (1994), 'Tubularized, incised plate urethroplasty for distal hypospadias', J Urol 151(2), 464-5.

Wolffenbuttel, K., Wondergem, N., Hoefnagels, J., Dieleman, G., Passchier, B., de Jong, B., van Dijk, W. \& Kok, D. (2005), 'Abnormal urine flow in boys with distal hypospadias before and after correction.', submitted . 